\title{
Direct Observation of Electron-to-Hole Energy Transfer in CdSe Quantum Dots
}

\author{
E. Hendry, ${ }^{1,2, *}$ M. Koeberg, ${ }^{1,2}$ F. Wang, ${ }^{3}$ H. Zhang, ${ }^{4}$ C. de Mello Donegá, ${ }^{5}$ D. Vanmaekelbergh, ${ }^{5}$ and M. Bonn ${ }^{1,2}$ \\ ${ }^{1}$ FOM Institute for Atomic and Molecular Physics, Kruislaan 407, 1098 SJ, Amsterdam, The Netherlands \\ ${ }^{2}$ Leiden Institute of Chemistry, Leiden University, P.O. Box 9502, 2300 RA Leiden, The Netherlands \\ ${ }^{3}$ Departments of Physics and Electrical Engineering, Columbia University, 538 West 120th Street, New York, New York 10027, USA \\ ${ }^{4}$ Van't Hoff Institute for Molecular Sciences, University of Amsterdam, Nieuwe Achtergracht 129, 1018 WS, \\ Amsterdam, The Netherlands \\ ${ }^{5}$ Department of Condensed Matter and Interfaces, Debye Institute, Utrecht University, Princetonplein 5, \\ 3508 TA Utrecht, The Netherlands
}

(Received 19 September 2005; published 9 February 2006)

\begin{abstract}
We independently determine the subpicosecond cooling rates for holes and electrons in CdSe quantum dots. Time-resolved luminescence and terahertz spectroscopy reveal that the rate of hole cooling, following photoexcitation of the quantum dots, depends critically on the electron excess energy. This constitutes the first direct, quantitative measurement of electron-to-hole energy transfer, the hypothesis behind the Auger cooling mechanism proposed in quantum dots, which is found to occur on a $1 \pm 0.15 \mathrm{ps}$ time scale.
\end{abstract}

DOI: 10.1103/PhysRevLett.96.057408

Semiconductor quantum dots (QDs) exhibiting strong quantum confinement of electrons provide a rare opportunity to study the fundamental properties of electronic excitations in a size regime between the atomic and bulk limits. Electron confinement gives rise to discrete energy levels [with $\left(1 S_{e}, 1 P_{e}, \ldots\right)$ electron and $\left(1 S_{3 / 2}, 1 P_{3 / 2}, \ldots\right)$ hole states] [1], rather than the continuum of states present in bulk semiconductors. In both bulk and QD semiconductors, absorption of a photon generates "hot" charges with energies in excess of the band edge. While in bulk materials cooling occurs readily through sequential one-phonon emission, the electron energy level spacing in QDs (typically hundreds of $\mathrm{meV}$ ) is large compared to typical longitudinal optical phonon frequencies $(\sim 25 \mathrm{meV})$, and electron cooling via coupling to phonons is expected to be slow. Accordingly, it has been proposed that cooling in QDs is hindered by a so-called "phonon bottleneck" [2], though cooling rates comparable to those in bulk have been observed [3-6], suggesting that other effects may prevail. The measurement and understanding of the decay dynamics of the different electron states is also of technological importance: QDs are increasingly finding applications as the active component in single-photon emitters, lightemitting diodes [7], photovoltaic cells [8,9], lasers [10], and photon up-converters; for all these applications, knowledge and manipulation of the decay dynamics of the hot and cold electron-hole (exciton) states is a prerequisite.

In the prototypical case of CdSe QDs, the radiative lifetime of the lowest exciton $1 S_{3 / 2} 1 S_{e}$ cold state has been studied extensively by time-resolved luminescence measurements [11]. However, when the hot $1 P_{3 / 2} 1 P_{e}$ exciton is generated through optical excitation, this state is not observed in the emission spectrum [1,11]. This means that nonradiative processes, i.e., electron cooling $\left(1 P_{e} \rightarrow\right.$ $\left.1 S_{e}\right)$ and hole cooling $\left(1 P_{3 / 2} \rightarrow 1 S_{3 / 2}\right)$, compete effec-
PACS numbers: 78.67.Hc, 65.80.+n, 73.21.La, 73.22.Dj

tively with radiative decay of the hot exciton. Thus, fast electron and/or hole cooling processes must occur, in contradiction with the predicted phonon bottleneck. To explain the apparent fast cooling, an Auger-like mechanism has been proposed [5,6,12-14], in which efficient transfer of energy from electrons to holes occurs followed by relatively fast hole relaxation through the more closely spaced valence levels [15]. Other explanations for rapid electron cooling include fast multiphonon relaxation [16] and polaron effects [17].

Most experimental studies to date have concentrated on the dynamics of the electron cooling through optical transient absorption measurements [3,4]. However, transitions in the optical regime correspond to interband transitions and are per definition determined by the dynamics of both electrons and holes. $1 P_{e}$ to $1 S_{e}$ electron relaxation has been studied with transient techniques using infrared pulses. In particular, Refs. [5,6] demonstrate that electron relaxation is slowed on modification of the QD surface, which is postulated to induce hole trapping. While such a link between the relaxation of electrons and holes is strong evidence of an Auger cooling mechanism, a definitive proof of electron-to-hole energy transfer requires independent determination of the hole relaxation dynamics. However, the lower energy hole transitions (which lie in the far-infrared [18]) are much more difficult to access with sufficient time resolution, and there have been no investigations to date on the dynamics of hole intraband transitions in CdSe QDs. A direct and independent determination of electron and hole cooling rates in single exciton QDs, which is required to unequivocally verify the Auger process and determine its cross section (i.e., to quantify the electron-hole interaction), has therefore remained elusive.

In this Letter, we determine the hole cooling dynamics in well-defined CdSe QD ensembles using terahertz time- 
domain spectroscopy (THz-TDS) [19-21]. The THz frequency range is sufficiently low that the response is dominated by hole transitions [21], and we observe subpicosecond hole cooling. Using this novel technique combined with femtosecond time-resolved photoluminescence [22], we directly observe electron-to-hole energy transfer, allowing a quantitative determination of electron-hole coupling time in CdSe QDs which compares very favorably to recent theoretical estimates.

Low-defect QD samples with small size dispersion $(\sim 5 \%)$ and high luminescence quantum yields $(50 \%-$ $90 \%$ ) are prepared by a modified version [11] of the high-temperature organometallic synthesis first developed by Talapin et al. [23], with QD size varying from a mean diameter $(D) \sim 1.7 \mathrm{~nm}$ to $D>10 \mathrm{~nm}$. Quantum confinement is expected to be negligible for $D>10 \mathrm{~nm}$. Both the average diameters and size distributions of all samples with $D<10 \mathrm{~nm}$ were obtained from the peak of the luminescence spectrum and its full width at half maximum (FWHM), respectively, following Ref. [11], in good agreement with transmission electron microscopy [11] performed on 4 samples. The measured dynamics were found to be intrinsic to the QDs and independent of the solvent (hexane or toluene). The $400 \mathrm{~nm}$ excitation fluence of $\sim 2 \mathrm{~J} \mathrm{~m}^{-2}$ is sufficiently low to ensure a maximum of one exciton per QD. All experiments were carried out at room temperature $(\sim 300 \mathrm{~K})$.

Exciting the QD samples with $400 \mathrm{~nm}(3.15 \mathrm{eV})$ photons leads to a distribution of hot electron and hole states, and information about the combined electron and hole cooling rates is obtained from the time evolution of luminescence from the $1 S_{3 / 2} 1 S_{e}$ (cold) state at the peak of the luminescence spectrum shown in the left panel in Fig. 1(a), determined with femtosecond (fs) luminescence up-conversion [24]. The luminescence signal is proportional to the product of hole and electron cold state populations. The results are plotted in the right-hand panel in Fig. 1(a).

The dynamics can be adequately described by a convolution between a measurement response function $G(\tau)$ (an independently determined Gaussian function with FWHM $\sim 560 \mathrm{fs}$ ) and an exponential rise described by time constant $\tau_{\text {rise }}$ :

$$
\int_{0}^{\infty} N_{0} \frac{\tau_{\text {decay }}}{\tau_{\text {decay }}-\tau_{\text {rise }}}\left(e^{-\tau / \tau_{\text {decay }}}-e^{-\tau / \tau_{\text {rise }}}\right) G\left(\tau^{\prime}-\tau\right) d \tau^{\prime} .
$$

A decay term $\left(\tau_{\text {decay }}\right)$ is included, as some hole trapping occurs in the smaller particles [21], quenching the luminescence. It should be noted that, since cooling and trapping occur on very different time scales, including trapping effects in the analysis does not significantly affect the derived rise times and simply allows a more accurate description of the data on longer time scales. The results of the fits are plotted as black lines in the right panel in Fig. 1(a). The extracted rise times are plotted (open circles) as a function of QD diameter $D$ in Fig. 2. (a) Luminescence

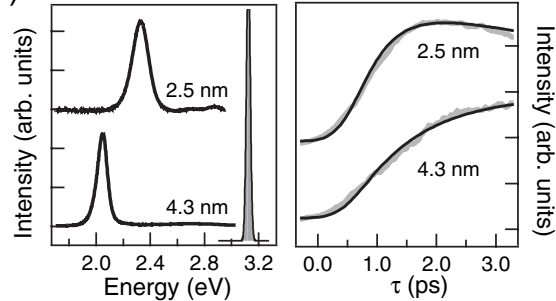

(b) THz-TDS

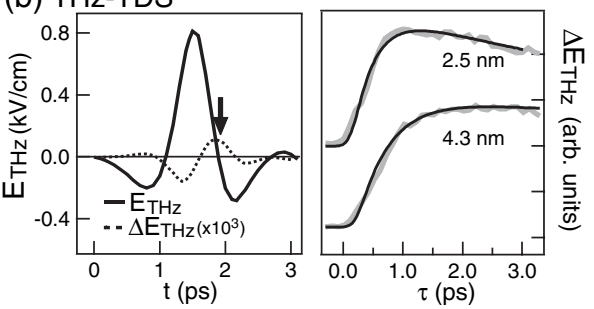

FIG. 1. (a) Left: Luminescence spectra. Also shown is the excitation pulse (shaded area). Right: We measure the increase in intensity at the peak of the luminescence spectra (gray lines) as a function of delay $\tau$. (b) Left: The transmitted THz pulses $E_{\mathrm{THz}}(t, \tau)$ and the exciton-induced modulation thereof, $\Delta E_{\mathrm{THz}}(t, \tau)$. Right: The modulation is measured at the point marked with an arrow in the left panel ( $t=1.9 \mathrm{ps})$, as a function of $\tau$, giving rise to the transient hole population of the $1 S_{3 / 2}$ level. The dynamics in both (a) and (b) are adequately described by exponential rise times determined by the carrier cooling rates (black lines in the right-hand panels).

There is a clear slowing down of the luminescence rise time for $D>3.3 \mathrm{~nm}$. These results can be understood by considering the energy of the $1 P_{3 / 2} 1 P_{e}$ transition [1] (the lowest energy, optically allowed transition for which hot electrons are photogenerated). For the fixed excitation energy used in the experiments, excitation of the $1 P_{3 / 2} 1 P_{e}$ and higher energy (hot) electron transitions are allowed only for QDs with $D>3.3 \mathrm{~nm}$. This means that for $D<3.3 \mathrm{~nm}$ the electron is always excited into the $1 S_{e}$ cold state, and hole cooling is solely responsible for the luminescence dynamics. For $D>3.3 \mathrm{~nm}$, the luminescence signal rise is slowed, owing to electron relaxation from the $1 P_{e}$ to the $1 S_{e}$ state. A very similar slowing of dynamics has also been observed in the $1 S_{3 / 2} 1 S_{e}$ absorption bleach for $D>3.3 \mathrm{~nm}$ [see Fig. 7(b) in Ref. [4]) but was interpreted as change in electron cooling, rather than the onset.

Though such fast dynamics indicate that a phonon bottleneck clearly does not limit the cooling, luminescence and optical transient absorption measurements do not offer direct proof of Auger-assisted cooling. This requires observation of electron-to-hole energy transfer. We investigate hole cooling using THz-TDS [19]. THz-TDS uses a weak electromagnetic field $(\sim 1 \mathrm{kV} / \mathrm{cm}) E_{\mathrm{THz}}(t, \tau)$, measured in the time $(t)$ domain following photoexcitation at time $\tau$, to probe the sample response in the range 0.1$8 \mathrm{meV}$. Such low energies are smaller than any energy level 


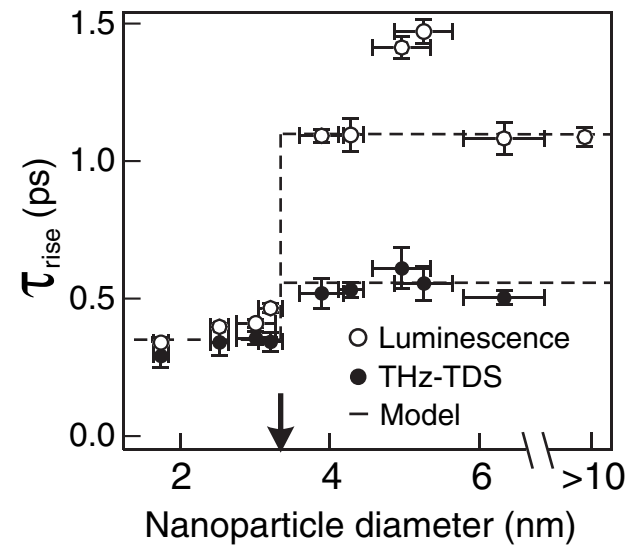

FIG. 2. Cooling times (describing the return to the cold electron and hole states) obtained from exponential fits to the $\mathrm{THz}-$ TDS and luminescence measurements as a function of mean particle diameter (horizontal error bars indicate size distributions). The dashed lines are the result of the model described in the text. The arrow marks the diameter for which (given the excitation energy) hot electron excitation is allowed, calculated from the energy levels in Ref. [1]-i.e., only for diameters $D \gtrsim$ $3.3 \mathrm{~nm}$ must electron (as well as hole) cooling be considered, marking a clear step in the luminescence and $\mathrm{THz}$ rise times in the main figure.

spacing in our QDs, and the signal is determined by the nonresonant response of the intraband transitions (i.e., the exciton polarizability $\alpha$ ) [20]. In particular, the $\alpha_{1 S_{3 / 2}}$ (lowest energy) hole state polarizability dominates the signal $[21,25]$. The polarization per unit volume $P$ induced by the probe $\mathrm{THz}$ field $E_{\mathrm{THz}}$ is described by

$$
P(t, \tau) \propto \int_{-\infty}^{t} N_{1 S_{3 / 2}}\left(t^{\prime}+\tau\right) E_{\mathrm{THz}}\left(t^{\prime}\right) \alpha_{1 S_{3 / 2}}\left(t-t^{\prime}\right) d t^{\prime},
$$

where $N_{1 S_{3 / 2}}$ is the density of holes in the $1 S_{3 / 2}$ state. For small changes to the incident $\mathrm{THz}$ field, the pump-induced change in the transmitted field is give by $\Delta E_{\mathrm{THz}}(t, \tau) \propto$ $\partial P(t, \tau) / \partial t$ [26]. Since the interaction of the exciton with the THz field is nonresonant, $\alpha_{1 S_{3 / 2}}(t)$ can be approximated by a delta function, and this simplifies to

$$
\begin{aligned}
\Delta E_{\mathrm{THz}}(t, \tau) \propto & \frac{\partial N_{1 S_{3 / 2}}(t+\tau)}{\partial t} E_{\mathrm{THz}}(t)+N_{1 S_{3 / 2}}(t+\tau) \\
& \times \frac{\partial E_{\mathrm{THz}}(t)}{\partial t} .
\end{aligned}
$$

Measuring $\Delta E_{\mathrm{THz}}(\tau)$ at a time $t$ when $E_{\mathrm{THz}}=0$ [marked by an arrow in Fig. 1(b)], the signal is entirely determined by the temporal dynamics of $N_{1 S_{3 / 2}}$, i.e., the transient hole population of the $1 S_{3 / 2}$ level. The experimentally observed dynamics are well described by Eq. (1) with $G(\tau)$ FWHM $\sim 215$ fs-see black lines in the right-hand panel in Fig. 1(b).

For QDs with $D<3.3 \mathrm{~nm}$, the $\mathrm{THz}$ rise times follows the luminescence rise times very closely $(\sim 0.3 \mathrm{ps}$; see
Fig. 2), suggesting that both are determined by hole cooling. For $D>3.3 \mathrm{~nm}$, there is a clear slowing of the luminescence rise (to $\sim 1.2 \mathrm{ps}$ ), indicating that electron cooling determines the delayed luminescence in larger particles. Remarkably, it is evident from the THz-TDS rise times that the hole cooling rate also slows down for $D>3.3 \mathrm{~nm}$, despite the fact that the hole itself has less excess energy when the electron is excited to the $1 P_{e}$ rather than the $1 S_{e}$ state. This observation is stressed in Fig. 3, which shows hole cooling times inferred from $\mathrm{THz}$ data as a function of hole excess energy, for electronic excitation into the cold $1 S_{e}$ and hot $1 P_{e}$ states. These relaxation times describe the rate at which the lowest energy hole state is occupied, limited by the slowest (final [27]) step in the overall relaxation process.

These observations allow for two conclusions: First, the rate of carrier cooling in CdSe QDs is clearly comparable to bulk cooling (indicated by the luminescence rise of the sample with $D>10 \mathrm{~nm}$ ), corroborating that a mechanism is available in which the phonon bottleneck is bypassed. Second, the observation of delayed hole cooling upon higher electronic excitation reveals that this mechanism entails energy transfer from electrons to holes (the holes are reexcited upon electron cooling, resulting in an apparent slowing down of hole cooling) and is the first direct confirmation of electron-to-hole energy transfer and the Auger cooling mechanism. The observed $\sim 1$ ps electronhole coupling time is in good agreement with theoretical predictions that range from 500 fs [13] to 2 ps [14]. An additional small increase $(\sim 0.25 \mathrm{ps})$ in the luminescence rise time for $D=4.5$ to $5 \mathrm{~nm}$, similar to that observed with

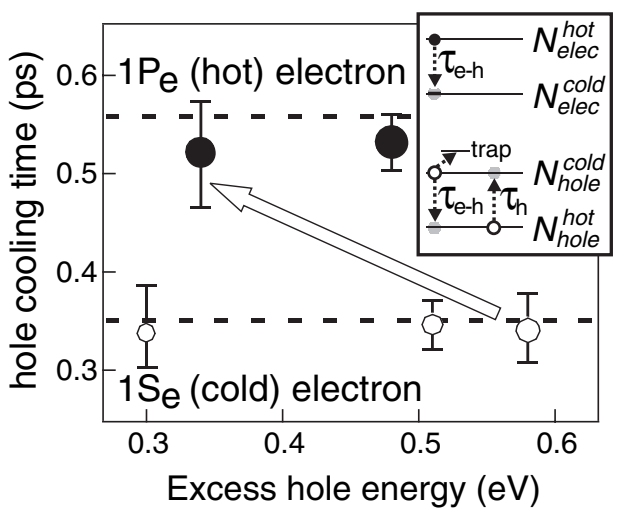

FIG. 3. Hole cooling time as a function of hole excess energy (calculated using the transitions in Ref. [1]), for QDs with diameters proportional to the symbol size $(2.5,3.0,3.2,3.9$, and $4.3 \mathrm{~nm}$, respectively). Solid symbols denote particles for which the electron $1 P_{e}$ state is optically accessible with the $3.15 \mathrm{eV}$ excitation pulse; open symbols are those for which the electron is excited into its $1 S_{e}$ state. The arrow indicates the transition occurring at $D \sim 3.3 \mathrm{~nm}$ : Hole cooling slows down considerably when the electron is excited into the hot state. The dashed lines are the result of the model. Inset: Schematic representation of the five-level model described in text. 
transient absorption measurements in Ref. [4], may be attributed to either electron cooling from higher lying excited states down to the $1 P_{e}$ state or slowing of the $1 P_{e} \rightarrow 1 S_{e}$ transition. As there must be a match to the energy separation between hole states involved in the Auger process (in order to conserve energy), one may expect the energy separation between $1 P_{e}$ and $1 S_{e}$ states (which varies with diameter [1]) to strongly affect Auger rates. However, when phonon interactions are included in the calculations in both Refs. $[13,14]$ the effects of energy level matching are smeared out, leading to electron-hole coupling times only weakly dependent on QD diameter and accounting for our modest size dependence of the luminescence rise time for $D>3.3 \mathrm{~nm}$.

To establish that the step in the hole cooling around $D=$ $3.3 \mathrm{~nm}$ can be quantitatively accounted for by electron-tohole energy transfer, we have modeled the system with a five-level calculation. Hot electron and hole states are represented by one level each (probabilities of occupation $N_{\text {elec }}^{\text {hot }}$ and $N_{\text {hole }}^{\text {hot }}$, respectively), as are the cold states (described by $N_{\text {elec }}^{\text {cold }}$ and $N_{\text {hole }}^{\text {cold }}$ )- -see inset in Fig. 3. The transitions between these levels are described by the hole cooling rate $\left(1 / \tau_{h}\right)^{-1}$ and the electron-hole coupling rate $\left(1 / \tau_{e-h}\right)^{-1}$. For $D<3.3 \mathrm{~nm}$, only cold electrons are produced upon photoexcitation, along with hot holes. Relaxation of hot holes leads to population of the cold state holes, from which the trapping rate $\left(1 / \tau_{\text {decay }}\right)^{-1}$ is again included for generality [21]. For $D>3.3 \mathrm{~nm}$, hot electrons are also produced, and electron relaxation is accompanied by the generation of hot holes (i.e., the Auger cooling mechanism). Numerical solution to this model [28] fully reproduces all observed transients for $D<4.5 \mathrm{~nm}$ (the diameter at which states higher than $1 P_{e}$ are excited, and more levels need to be included for an accurate description) with $\tau_{h}=330 \pm 50 \mathrm{fs}$ and $\tau_{e-h}=$ $1 \pm 0.15$ ps. The latter is in good agreement with both theoretical estimates of the electron-hole coupling time $[13,14]$ and with the $1 P_{e} \rightarrow 1 S_{e}$ electron relaxation experimentally determined by infrared pump-probe spectroscopy [5]. The calculated signals [proportional to $N_{\text {hole }}^{\text {cold }}(\mathrm{THz})$ and $N_{\text {elec }}^{\text {cold }} \times N_{\text {hole }}^{\text {cold }}$ (luminescence), convoluted with the corresponding measurement response function] can be described very well using Eq. (1), and the derived exponential times are shown as dashed lines in Fig. 2. Clearly, the increase in hole cooling time for $D>3.3 \mathrm{~nm}$ can be fully accounted for by electron-to-hole energy transfer.

The observed Auger-assisted cooling has important implications for the application of QDs in optoelectronics. Such fast cooling is favorable for QD lasers [10], while it is detrimental for the harvesting of hot charges in QD solar cells [29]. Harvesting carriers before cooling requires either extremely rapid, subpicosecond charge collection or the suppression of Auger effects through hole trapping and/ or designing QDs with similar electron and hole energy level spacing.

This work is part of the research program of the "Stichting voor Fundamenteel Onderzoek der Materie (FOM)," which is financially supported by the "Nederlandse organisatie voor Wetenschappelijk Onderzoek (NWO)."

*Electronic address: hendry@amolf.nl

[1] D. J. Norris and M. G. Bawendi, Phys. Rev. B 53, 16338 (1996).

[2] A. J. Nozik, Annu. Rev. Phys. Chem. 52, 193 (2001).

[3] V. I. Klimov and D. W. McBranch, Phys. Rev. Lett. 80, 4028 (1998).

[4] V. I. Klimov et al., Phys. Rev. B 60, 13740 (1999).

[5] P. Guyot-Sionnest et al., Phys. Rev. B 60, R2181 (1999).

[6] V. I. Klimov et al., Phys. Rev. B 61, R13 349 (2000).

[7] V. L. Colvin et al., Nature (London) 370, 354 (1994).

[8] W. U. Huynh et al., Science 295, 2425 (2002).

[9] E. W. McFarland and J. Tang, Nature (London) 421, 616 (2003).

[10] V. I. Klimov et al., Science 290, 314 (2000).

[11] C. d. M. Donegá et al., J. Phys. Chem. B 107, 489 (2003).

[12] V. I. Klimov et al., Science 287, 1011 (2000).

[13] L. W. Wang et al., Phys. Rev. Lett. 91, 056404 (2003).

[14] A. L. Efros et al., Solid State Commun. 93, 301 (1995).

[15] S. Xu et al., Phys. Rev. B 65, 045319 (2002).

[16] P. C. Sercel, Phys. Rev. B 51, 14532 (1995).

[17] S. Sauvage et al., Phys. Rev. Lett. 88, 177402 (2002).

[18] A. L. Efros and M. Rosen, Annu. Rev. Mater. Sci. 30, 475 (2000).

[19] M. C. Beard et al., J. Phys. Chem. B 106, 7146 (2002).

[20] E. Hendry et al., Phys. Rev. Lett. 92, 196601 (2004).

[21] F. Wang et al. (to be published).

[22] D. F. Underwood et al., J. Phys. Chem. B 105, 436 (2001).

[23] D. V. Talapin et al., Nano Lett. 1, 207 (2001).

[24] P. v. d. Meulen et al., J. Phys. Chem. 100, 5367 (1996).

[25] A simple estimate for the polarizability $(\alpha)$ of states in a QD can be obtained by considering simple particle-in-abox-type solutions to the Schrödinger equation $\left(\psi_{n}\right.$, with eigenenergy $E_{n}$ [see S. V. Gaponenko, Optical Properties of Semiconductor Nanocrystals (Cambridge University Press, Cambridge, England, 1998)]) using $\alpha=\frac{2}{3} \times$ $\left(1 / 4 \pi \varepsilon_{0}\right) \sum \psi_{\text {final }}\left|\left\langle\psi_{\text {initial }}|e \tilde{r}| \psi_{\text {final }}\right\rangle\right|^{2} /\left(E_{\text {final }}-E_{\text {initial }}\right)$. From this, we estimate hole polarizabilities 3 orders of magnitude larger than those for electron states. The lowest energy hole state contributes a factor 9 times more than the first hot state. See also Ref. [21].

[26] E. Hendry et al., Phys. Rev. B 71, 125201 (2005).

[27] H. Htoon et al., Phys. Rev. Lett. 93, 187402 (2004).

[28] E. Hendry, Ph.D. thesis, University of Amsterdam, 2005.

[29] A. J. Nozik, in Proceedings of the 2003 NCPV and Solar Program Review Meeting, Denver, CO (National Renewable Energy Laboratory, Washington, D.C., 2003), p. 422. 